Orange Journal / Volumen 2 Número 4/ Julio - diciembre 2020

DOI: https://doi.org/10.46502/issn.2710-995X/2020.4.04

\title{
Percepción de la comunicación del farmacéutico desde farmacias comunitarias del municipio Santiago de Cuba
}

\author{
Perception of the pharmacist's communication from community pharmacies of the \\ Santiago de Cuba municipality
}

Aceptado: 5 de mayo de 2021

Escrito por:

Alicia Aldana Laterrade ${ }^{9}$

https://orcid.org/0000-0001-8474-3941

Rosario Megret Despaigne ${ }^{10}$

https://orcid.org/0000-0003-1925-9795

Maislin Garzón Feria ${ }^{11}$

https://orcid.org.0000-0001-7648-0459

Elinay Brugal Mustelier ${ }^{12}$

https://orcid.org.0000-0002-3163-2479

\section{Resumen}

La comunicación activa del farmacéutico garantiza la adherencia terapéutica y la satisfacción del paciente. Se realizó un estudio del tipo observacional, descriptivo y transversal con el objetivo de evaluar la percepción de la comunicación del farmacéutico desde cinco farmacias comunitarias del Municipio Santiago de Cuba, en el segundo semestre de 2019. La muestra estuvo conformada por 36 farmacéuticos, cumpliéndose las normas éticas. Como instrumento de trabajo se utilizó un cuestionario aplicado en el puesto laboral, los profesionales fueron caracterizados atendiendo a categoría ocupacional, años de práctica profesional y superación, valorándose los criterios de Recursos, Actitud y Visión, Formación y Regulatorio y Ambiente. Se determinó el nivel de percepción en favorable, poco favorable y desfavorable. Se realizó un análisis porcentual de los datos determinándose media y desviación estándar de los criterios. Los resultados demostraron que el 55,55\% se desempeñó como Dependientes de Farmacia, seguido de los Licenciados en Ciencias Farmacéuticas y en Tecnología de la Salud que representaron el 33,33\% y por último los Técnicos en Farmacia (11,11\%). Existió mayor prevalencia entre 6-12 años de experiencia profesional para un 52,78\%, se halló que solo el 5,55\% ha continuado sus estudios de formación posgraduada a nivel de maestría. Resultaron poco favorable los criterios: Recursos y Regulatorio y ambiente, mientras que Actitud y visión y Formación fueron favorables. La percepción resultó favorable para un

\footnotetext{
9 Directora UEB Distrito 2. Empresa Provincial Farmacias y Ópticas. Santiago de Cuba.

${ }^{10}$ Departamento de Farmacia. Facultad de Ciencias Naturales y Exactas. Universidad de Oriente.

${ }^{11}$ Farmacia Comunitaria Edificio Sueño. Empresa Provincial de Farmacias y Ópticas. Santiago de Cuba.

${ }^{12}$ Farmacia Comunitaria Bloque B. Empresa Provincial de Farmacias y Ópticas. Santiago de Cuba.
} 
$55,55 \%$, seguida de la poco favorable en $30,55 \%$ y desfavorable un $13,88 \%$. Los farmacéuticos percibieron como favorable la comunicación con el paciente en las farmacias comunitarias estudiadas.

Palabras claves: comunicación, farmacéutico, farmacia comunitaria, percepción.

\begin{abstract}
Active communication from the pharmacist ensure therapeutic adherence and patient satisfaction. An observational, descriptive and cross-sectional study was carried out with the objective of evaluating the perception of the pharmacist's communication from community pharmacies of the Santiago de Cuba Municipality, in the second semester of 2019. The sample consisted of 36 pharmacists, complying with the norms ethical. As a work instrument, a questionnaire applied in the workplace was used, the professionals were characterized according to occupational category, years of professional practice and improvement, assessing the criteria of Resources, Attitude and vision, Training and Regulatory and environment and it was determined the level of perception in favorable, unfavorable and unfavorable. A percentage analysis of the data was performed, determining the mean and standard deviation of the criteria. The results showed that $55.55 \%$ worked as Pharmacy Dependents, followed by Graduates in Pharmaceutical Sciences and in Health Technology who represented $33.33 \%$ and finally Pharmacy Technicians (11.11\%). There was a higher prevalence between 6-12 years of professional experience for $52.78 \%$, it was found that only $5.55 \%$ have continued their postgraduate training studies at the master's level. The criteria were unfavorable: Resources and Regulatory and environment, while Attitude and vision and Training were favorable. The perception was favorable for $55.55 \%$, followed by unfavorable $30.55 \%$ and unfavorable $13.88 \%$. Pharmacists perceived communication with the patient as favorable in the community pharmacies studied.
\end{abstract}

Keywords: communication, community pharmacy, perception, pharmacist.

\title{
Introducción
}

La farmacia comunitaria es el local de salud pública más visitado por el paciente, es donde se desarrollan las funciones asistenciales de dispensación, educación sanitaria, indicación farmacéutica, seguimiento farmacoterapéutico y farmacovigilancia que propician una asistencia completa al paciente ambulatorio (Martínez, 2015).

En la labor asistencial del farmacéutico, como en cualquier profesión sanitaria, existe un proceso comunicativo, esta comunicación constituye un instrumento para alcanzar los objetivos planteados en los servicios farmacéuticos a través de una información adecuada sobre la medicación para asegurar su utilización correcta (Martín y Valles, 2014).

Las competencias adquiridas en cuanto a comunicación y su perfeccionamiento mejoran la actividad asistencial en la farmacia comunitaria en función de sus responsabilidades, verificando los aspectos necesarios para el cumplimiento terapéutico por parte del 
paciente y las mejoras en su calidad de vida, además de promocionar salud y prevenir enfermedad (Coca, 2018 y González, 2019).

La percepción es considerada uno de los elementos sociales y psicológicos de la comunicación, refleja un proceso a través del cual poseemos conciencia de lo que ocurre a nuestro alrededor y en nosotros mismos; el individuo interpreta la información de acuerdo a experiencias previas, estas se organizan en forma lógica en el mismo acto de observación de una cosa, la organización perceptiva es consecuencia de los procesos físicos que tienen lugar en la corteza cerebral (Ventocilla, 2015)

En la actualidad diversas investigaciones exponen el tema de la percepción de la comunicación farmacéutico-paciente, donde se demuestra el carácter reactivo de este profesional; las habilidades comunicacionales necesarias para realizar la Atención Farmacéutica; la comunicación participativa e interpersonal en la calidad de la relación y en la satisfacción del paciente; así como las barreras que influyen en este proceso, fundamentalmente la falta de espacio, de personal y de colaboración con otros profesionales(Valles et al., 2018; Amibor et al., 2019 y Prasojo et al., 2019).

Partiendo del análisis de estos estudios, que reflejan la complejidad del acto de comunicación del farmacéutico con el paciente, la presencia de barreras en el proceso comunicativo, que las investigaciones se realizan mayoritariamente desde la perspectiva del paciente, en contextos hospitalarios y que en Cuba existen escasos estudios que documentan la apreciación de este proceso desde el punto de vista del profesional; en este trabajo nos propusimos evaluar la percepción de la comunicación del farmacéutico desde Farmacias Comunitarias del Municipio Santiago, Provincia Santiago de Cuba.

\section{Marco teórico}

Farmacia Comunitaria: es una entidad de salud donde los farmacéuticos desarrollan actividades destinadas al uso de los medicamentos, a través de la dispensación, preservando el cumplimiento de la prescripción de los profesionales sanitarios con quien, además, cooperarán en el servicio de Seguimiento Farmacoterapéutico de acuerdo a los procedimientos de la Atención Farmacéutica, logrando la eficacia y seguridad de los medicamentos. Por tanto, el farmacéutico comunitario asegura un servicio asistencial en contacto directo con pacientes y usuarios como parte de los eslabones de adquisición de los productos farmacéuticos (Sedeño y Sánchez, 2020).

Comunicación: es el acto de intercambiar información entre un emisor y un receptor, esta puede ser unidireccional o bidireccional. Las formas de comunicación se dividen en dos: la comunicación verbal que se representa a través de las palabras y a los tonos de la voz y la comunicación no verbal que hace referencia a los mensajes no verbales como la expresión corporal, gestos, manera de vestir, contacto visual y la distancia corporal (Choque, 2005).

El proceso de comunicación constituye un elemento fundamental en los sistemas y servicios de salud como parte de las acciones de prevención y promoción de esta, que debe instituirse como una estrategia básica para lograr la información a la población sobre 
aspectos referentes a la salud. Una estrategia de comunicación centrada en el paciente, es conocer de las personas su personalidad, antecedentes patológicos/ familiares y su espectro social, para desarrollar una comprensión compartida del problema de salud y los objetivos del tratamiento (Sabater et al., 2016).

Relación farmacéutico-paciente: El farmacéutico comunitario es el profesional sanitario que mejor puede contribuir a garantizar el uso seguro de los medicamentos debido a la cercanía y accesibilidad de los usuarios con la farmacia. El desarrollo de servicios asistenciales en la farmacia comunitaria ha conllevado a una tendencia creciente en la relación entre el farmacéutico y el paciente (Sabarte,2016).Varias investigaciones en la relación farmacéutico-paciente han sido desarrolladas desde diferentes perspectivas, incluyendo los impactos en la calidad de la relación, satisfacción del paciente, resultados de salud o modificación del comportamiento frente a hábitos no saludables ( Wells et al., 2014; Pérez y Valdearcos,2018)

\section{Metodología}

\section{Tipo, lugar, período y diseño del estudio}

Se realizó un estudio observacional, descriptivo y transversal, en 36 farmacéuticos comunitarios del Municipio Santiago, Provincia Santiago de Cuba, durante el segundo semestre de 2019, con el objetivo de evaluar la percepción de la comunicación del farmacéutico desde farmacias comunitarias.

\section{Universo y muestra}

Se escogieron de forma aleatoria farmacéuticos que laboran en Farmacias Comunitarias Municipio Santiago: Principal Municipal Hospitalaria, Principal Distrital Las Américas, Normal Garzón 2, Normal Independencia, Normal Edificio Sueño y Normal Matagalpa, estas farmacias fueron seleccionadas por la cobertura farmacéutica, los servicios que brindan a la atención primaria, policlínicos, hospitales y su ubicación geográfica. Para obtener la muestra del universo de los farmacéuticos se establecieron los siguientes criterios de selección:

Criterios de inclusión: Farmacéuticos que prestan servicios de dispensación o que están responsabilizados con el Programa Nacional de Medicamentos, que ofrecieron su consentimiento de forma oral para participar en la investigación.

Criterios de exclusión: Farmacéuticos que prestan servicios de dispensación o que están responsabilizados con el Programa Nacional de Medicamentos, con menos de 5 años de experiencia laboral.

\section{Caracterización de la muestra objeto de estudio teniendo en cuenta las variables:}

Categoría ocupacional: Licenciado, Técnico Medio y Dependiente de Farmacia.

Años de práctica profesional: 6-12 años, 13-18 años, $\geq 20$ años. 
Superación: Posgrado, diplomado y/o Maestría.

\section{Determinación de la percepción de la comunicación}

Para la determinación de la percepción de la comunicación desde la perspectiva del farmacéutico, se utilizó como instrumento un cuestionario autoadministrado, validado por Álvarez et al. (2017) y adaptado a nuestras condiciones de trabajo. El cuestionario consta de 12 preguntas divididas en los siguientes criterios: I-Recursos (3 preguntas), II-Actitud y visión (3 preguntas), III- Formación (2 preguntas) y IV-Regulatorio y ambiente (4 preguntas). A cada respuesta se le dio una puntuación máxima de 5 puntos y se establecieron según el puntaje promedio, los siguientes niveles de percepción:

Percepción Favorable: > 3,5 puntos

Percepción Poco Favorable: 2,5-3,5 puntos

Percepción Desfavorable: $<2,5$ puntos

\section{Análisis estadísticos}

Se realizó un análisis descriptivo de los resultados con análisis porcentual y se calcularon la media y la desviación estándar, se confeccionaron tablas y figuras mediante herramientas de Microsoft Office 2013.

\section{Aspectos éticos}

Todos los farmacéuticos participaron en el estudio de forma voluntaria, siendo informados de los objetivos de la investigación. Se les garantizó el anonimato y la confidencialidad de la información. Este trabajo contó con la aprobación del Consejo Científico de la Empresa Provincial de Farmacias y Ópticas de Santiago de Cuba, acuerdo No 6/2018.

\section{Resultados}

\section{Caracterización de la muestra objeto de estudio}

En la investigación participaron 36 profesionales de las farmacias comunitarias seleccionadas, que cumplieron con los criterios de inclusión, la caracterización se aprecia en la tabla 1, donde el 55,55\% se desempeñó como Dependientes de Farmacia, seguido de los Licenciados en Ciencias Farmacéuticas y en Tecnología de la Salud con especialidad en Servicios Farmacéuticos que representaron el 33,33\% y por último los Técnicos Medios (11,11\%).

En cuanto a los años de práctica profesional existió mayor prevalencia entre 6-12 años para el $52,78 \%$ y en menor proporción $\geq 20$ años con el $16,67 \%$, para una media de 13,05 $\pm 5,02$. Referente a la superación se halló que solo el 5,55\% ha continuado sus estudios de formación posgraduada a nivel de maestría. 


\section{ORANGE JOURNAL}

Tabla 1.

Caracterización de la muestra objeto de estudio.

\begin{tabular}{|l|c|c|c|}
\hline \multicolumn{2}{|c|}{ Datos Generales } & $\mathrm{N}$ & $\%$ \\
\hline Categoría ocupacional & Licenciado & 12 & 33,33 \\
\hline & Técnico Medio & 4 & 11,11 \\
\hline Años de práctica & Dependiente & 20 & 55.55 \\
profesional & $6-12$ & 19 & 52,78 \\
\hline Superación & $13-19$ & 11 & 30,55 \\
& $\geq 20$ & 6 & 16,67 \\
& Posgrado & - & - \\
\hline Diplomado & - & - \\
\hline
\end{tabular}

$N$ : número de Farmacéuticos $=36$

Fuente: Cuestionario de investigación.

\section{Determinación de la percepción de la comunicación}

El criterio I exploró la percepción de los farmacéuticos acerca de los recursos necesarios para desarrollar la comunicación con los pacientes y usuarios de las farmacias, como se puede apreciar en la figura 1, el 77,77\% planteó no tener tiempo para ésta actividad; el $61,11 \%$ refiere la disponibilidad de fuentes de información que permiten la preparación del farmacéutico y el $66,66 \%$ respondió que la farmacia cuenta con espacio para atender a la población.

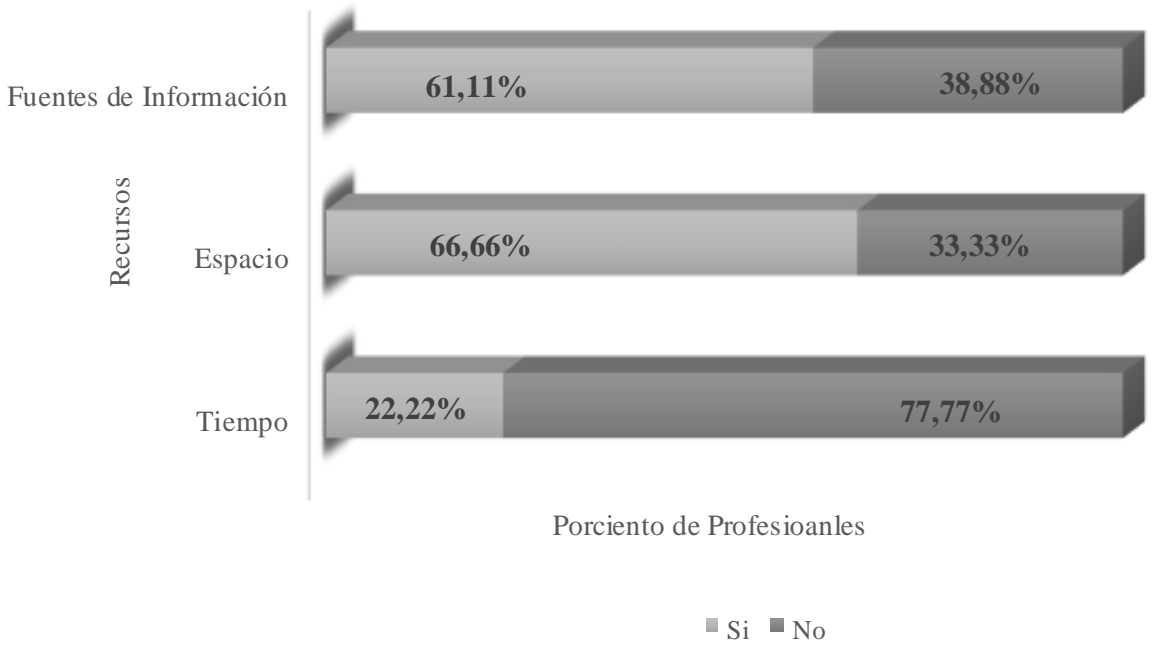

Figura 1. Respuestas de los farmacéuticos según criterio: Recursos.

Fuente: Elaborado por los autores.

En cuanto al criterio II-Actitud y visión (figura 2), se observa que la disposición de los farmacéuticos fue adecuada en el 80,58\%, así como la actitud de los empleadores para el 77,80\%; referente a la actitud de los pacientes, el 58,33\% de los farmacéuticos opinaron que es poco adecuada mientras que el 41,66\% apreció que es adecuada. 


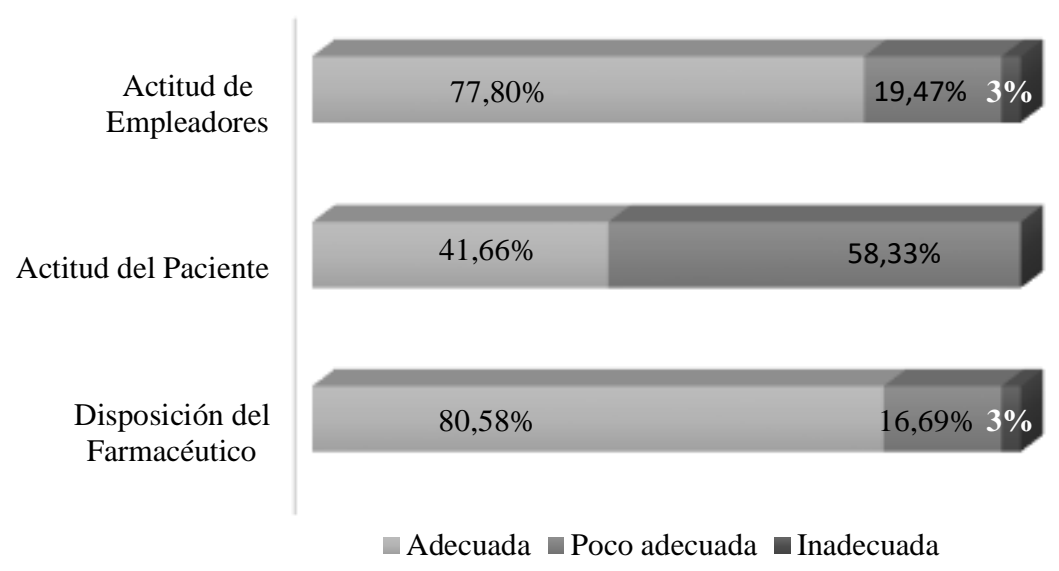

Figura 2. Respuestas de los farmacéuticos según criterio: Actitud y visión.

Fuente: Elaborado por los autores.

Al analizar el criterio III-Formación de pregrado y posgrado, respecto a los conocimientos y habilidades de la comunicación en farmacia, se muestra en la figura 3, como para la formación pregrado predominó la categoría Buena con el 72,22\% a diferencia del posgrado que obtuvo la categoría Regular con el $86,11 \%$.

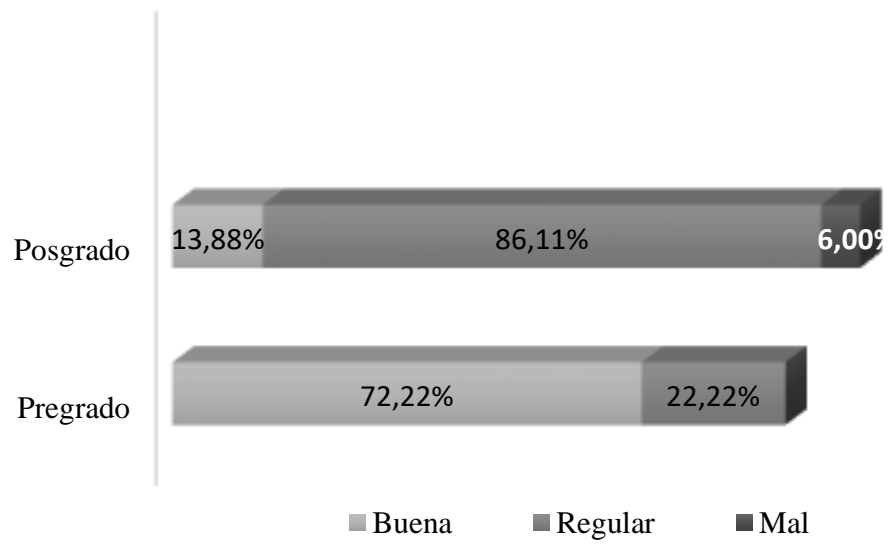

Figura 3. Respuestas de los farmacéuticos según criterio: Formación.

Fuente: Elaborado por los autores.

La figura 4 refleja los resultados del criterio IV-Regulatorio y ambiente, obteniéndose que el $100 \%$ de la muestra refirió que el proceso de comunicación ocupa un lugar importante para lograr la satisfacción del paciente; el 66,66\% planteó que existe un procedimiento que regula esta actividad dentro de la dispensación; un 55,55\% de los encuestados describieron que las condiciones ambientales que presenta la farmacia favorecen la comunicación y la interacción farmacéutico-paciente; mientras que el $55,55 \%$ respondieron que no se evalúan las actividades de comunicación con los pacientes. 


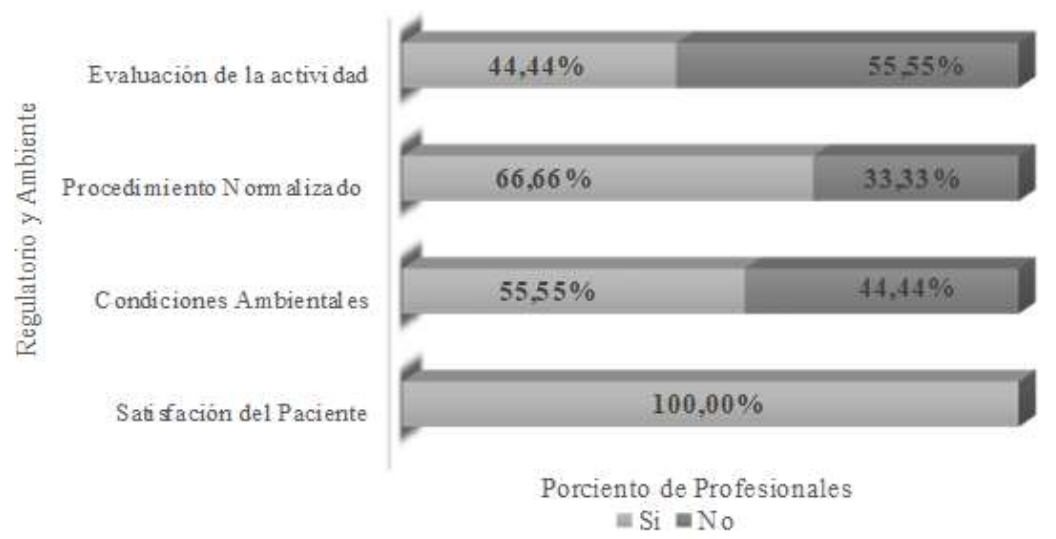

Figura 4. Respuestas de los farmacéuticos según criterio: Regulatorio y ambiente.

Fuente: Elaborado por los autores.

La percepción de cada criterio evaluado según la media aritmética de la puntuación, se expone en la tabla 2, en ella se describe que resultaron poco favorables los criterios: IRecursos y IV-Regulatorio y ambiente; los correspondientes a II-Actitud y visión y IIIFormación fueron favorables. En cuanto a la percepción global (figura5), se obtuvo que el 55,55\% de los farmacéuticos percibieron como favorable el proceso de comunicación, seguido del poco favorable para el $30,55 \%$ y desfavorable el $13,88 \%$.

\section{Tabla 2.}

Criterios según nivel de percepción.

\begin{tabular}{|l|c|c|}
\hline \multicolumn{1}{|c}{ Criterios } & $\mathrm{X}^{-} \pm \mathrm{DS}$ & Nivel de percepción \\
\hline I-Recursos & $2,50 \pm 1,21$ & Poco Favorable \\
\hline II-Actitud y visión & $4,20 \pm 0,39$ & Favorable \\
\hline III-Formación & $3,70 \pm 0,02$ & Favorable \\
\hline IV-Regulatorio y ambiente & $3,20 \pm 1,20$ & Poco Favorable \\
\hline
\end{tabular}

$X^{-}=$Media $\quad D S=$ Desviación Estándar

Fuente: Cuestionario de investigación.

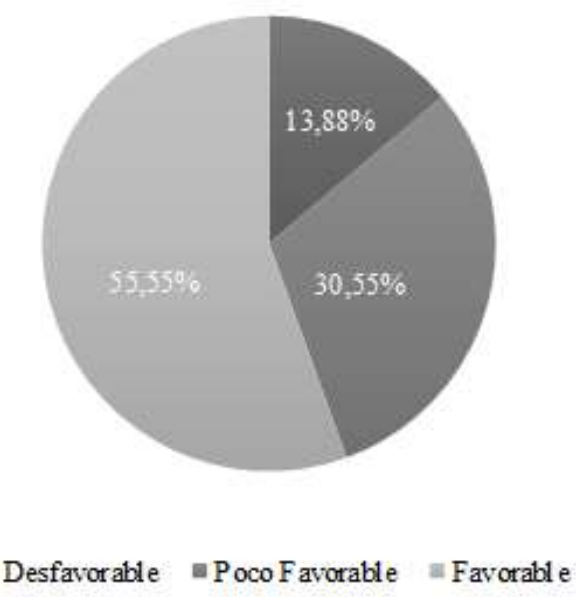

Figura 5. Percepcion global.

Fuente: Elaborado por los autores. 


\section{Discusión}

\section{Caracterización de la muestra objeto de estudio}

En relación a la variable categoría ocupacional los resultados muestran predominio de los farmacéuticos que se desempeñan como Dependientes de Farmacia, por su incidencia en la atención que le ofrece a la población este personal debe estar consciente de las funciones y responsabilidades que ha de asumir y de la importancia asistencial, económica, legal y ética que conllevan; resultados similares fueron encontrados en investigaciones desarrolladas en Cuba por Mateu et al. (2017) y Elías et al. (2019), donde se plantean que generalmente la atención a los pacientes la brindan los Dependientes de Farmacia.

En nuestro país la mayoría de los dependientes tienen varios años de experiencia en el sector con el requisito de poseer duodécimo grado y el certificado de habilitación otorgado por la Facultad de Tecnologías Médicas luego de un examen aplicado por esta misma institución, por lo que están preparados para recibir prescripciones médicas y realizar el expendio de medicamentos (Reglamento General de Farmacias Comunitarias, 2016).

En la muestra el 33,33\% presenta título de Licenciados en Ciencias Farmacéuticas y en Tecnología de la Salud con especialidad en Servicios Farmacéuticos quienes supervisan y controlan los procesos en las áreas de la farmacia, dadas las exigencias de trabajo este personal ha de ser capacitado y adiestrado en las funciones que asumirá.

El valor promedio de 13,05 $\pm 5,02$ años de experiencia puede influir adecuadamente en el desarrollo de las habilidades comunicativas verbal y no verbal, ya que estos profesionales han aplicado hasta el momento los conocimientos adquiridos en la formación y perfeccionado la práctica de dispensación. Resultados semejantes fueron hallados en otras investigaciones, donde la mayoría de los farmacéuticos comunitarios presentaban 10 años o más de experiencia profesional (García et al., 2016; Pérez y Valdearcos, 2018 y Lugo et al., 2019).

Estos resultados difieren de los encontrados por Pérez et al. (2019) en un estudio realizado en Salamanca con profesionales sanitarios responsables del suministro de medicamentos donde un $8 \%$ resultaron profesionales noveles (hasta 2 años de experiencia), un $38 \%$ con experiencia (3 a 10 años de experiencia) y un $54 \%$ a término de su vida laboral (11 o más años de experiencia).

\section{Determinación de la percepción de la comunicación}

$\mathrm{Al}$ analizar los criterios evaluados en la determinación de la percepción, se demostró que para desarrollar el proceso de comunicación a través de la dispensación son necesarios varios recursos como tiempo, bibliografía y espacio, representados en el criterio I de la investigación. Este resultado fue similar al descrito por Roig et al. (2012) que determinaron que dentro de los recursos precisos para la adecuada interacción entre farmacéuticos y usuarios se encuentran: el tiempo, un espacio para informar y educar, 
personal cualificado y un registro de la actividad; además de material de oficina, folletos, láminas y trípticos para ofrecer, lo que permiten una mayor apropiación de la información al paciente/usuario.

En cuanto al criterio II-Actitud y visión, la literatura señala que no sólo es importante la disposición por parte del farmacéutico, sino también de los empleadores y pacientes, que posibiliten el desarrollo adecuado del proceso de comunicación para que este profesional pueda ofrecer información acerca del uso correcto de los medicamentos, conservación y posibles reacciones adversas, que garantice la satisfacción de los pacientes y una mayor adherencia terapéutica. Las diferentes respuestas de los farmacéuticos en cuanto a la actitud mostrada por los pacientes pueden estar dada por los tipos de comportamientos de las personas, sus impaciencias y expectaciones ante la adquisición de sus medicamentos e insumos farmacéuticos.

En el criterio III-Formación, el pregrado fue evaluado de bueno, ya que los últimos planes de estudios para los licenciados han estado dirigido a garantizar la formación asistencial del egresado, teniendo en cuenta la tendencia de la Atención Farmacéutica enfocada a la prevención y detección de los problemas relacionados con los medicamentos; de esta forma se han incluido asignaturas como la Farmacoterapia, Atención Farmacéutica, Comunicación, Educación Sanitaria y Servicios Farmacéuticos que tributan a la adquisición de conocimientos y habilidades comunicativas para la provisión de los servicios profesionales farmacéuticos (Ministerio de Educación Superior. Cuba, 2017 y Suárez, 2017).

De acuerdo a la bibliografía revisada las facultades de farmacia necesitan introducir módulos que traten sobre los principios de la comunicación, oral y escrita, y ofrezcan métodos de aprendizaje y evaluación que permitan a los estudiantes adquirir y desarrollar esas aptitudes. Es esencial que todo el personal de salud esté cuidadosamente capacitado para llevar a cabo el proceso comunicativo de manera confiable y eficiente (Pérez et al., 2014).

En el estudio pocos son los profesionales que cuentan con cursos de posgrado, aun cuando el Departamento de Farmacia de la Universidad de Oriente, divulga su plan de posgrado y cuenta con la Maestría en Servicios Farmacéuticos que brinda una formación integral a los farmacéuticos de la región oriental.

En el criterio IV-Regulatorio y ambiente, los profesionales perciben que la comunicación es esencial para lograr la satisfacción de los pacientes, lo que constituye un indicador de calidad de las farmacias comunitarias; la satisfacción de los pacientes es uno de los resultados considerado por Choque et al. (2019), que plantean que brindar la mejor calidad del servicio a través de un trato amable, rápido y eficiente, logra una fidelización de los clientes.

En la respuesta a la existencia de un procedimiento que regule la actividad, más de la mitad de la muestra estudiada asegura contar con un procedimiento que rige la actividad de la Farmacia Comunitaria y como parte de este la normalización del proceso de dispensación, sin embargo es necesario que todos los servicios que se realizan en la 
farmacia dispongan de una guía práctica, por lo que recomendamos que en la actualización del Manual de Normas y Procedimientos de las Farmacias Comunitarias en Cuba se describan acciones específicas acerca de la comunicación farmacéutico-paciente dentro del servicio de dispensación con indicadores que permitan evaluar cada una de las actividades.

Las condiciones ambientales del área de dispensación están descritas en este manual, aunque las mismas no se cumplen en todas las farmacias estudiadas, ya que esta área debe tener la adecuada amplitud, higiene, ventilación e iluminación, considerando que estos factores contribuyen a una mejor protección de los medicamentos y una óptima circulación del personal, tanto de los propios trabajadores de la farmacia como de los usuarios que acuden a ella. Se procura la utilización de los diferentes espacios disponibles (mostradores, vidrieras, murales) para brindar información a pacientes y usuarios que acuden a la unidad (Ministerio de Salud Pública, 2005).

Al resumir la percepción de acuerdo con los criterios evaluados, encontramos que los criterios II-Actitud y visión y III- Formación resultaron favorables, mientras que los criterios I-Recursos y IV-Regulatorio y ambiente fueron desfavorables, lo que coincide con otros estudios que han demostrado que la falta de recursos y el ambiente inadecuado constituyen barreras en el proceso de comunicación farmacéutico-paciente (Saffouh et al., 2016 y Lemay et al., 2018).

Por último en relación a la percepción global de la comunicación con el paciente desde la perspectiva del farmacéutico comunitario prevaleció la favorable, aunque se mostraron los tres niveles de percepción; estos resultados pueden estar dado a que los farmacéuticos que participaron en el estudio pertenecen a farmacias comunitarias de diferentes clasificaciones según los servicios y grado de atención que brindan; por lo que los recursos, el entorno, las categorías ocupacionales así como la población atendida varían en cada una de estas, lo que conlleva a una apreciación distinta del proceso de comunicación.

El estudio presenta limitaciones por el número de farmacéuticos de solo seis farmacias comunitarias del municipio, no obstante, consideramos que la investigación es una aproximación a la evaluación de la percepción de la comunicación desde la perspectiva de este profesional. Por otro lado, la utilización de una escala numérica corta para la puntuación del cuestionario, puede generar confusión en el significado de las respuestas. Se considera realizar otras investigaciones aumentando el número de muestra.

\section{Conclusiones}

Los farmacéuticos que laboran en las Farmacias Comunitarias seleccionadas perciben que la comunicación con el paciente es favorable, teniendo en cuenta que han aplicado los conocimientos adquiridos en la formación y perfeccionado la práctica de dispensación desde la Atención Farmacéutica, enfocada en la prevención y detección de problemas relacionados con los medicamentos ofreciendo información sobre conocimientos y habilidades comunicativas en la provisión de los servicios profesionales farmacéuticos, además la actitud de los empleadores y pacientes, posibilitó el desarrollo adecuado del 
proceso de comunicación para la adecuada interacción entre farmacéuticos y usuarios acerca del uso correcto de los medicamentos, garantizando la satisfacción de los pacientes.

\section{Referencias Bibliográficas}

Álvarez, A., Yi L., Del Águila, S., \& Pei, Y. (2017). Barreras para la provisión de Atención Farmacéutica en farmacias de Tainan, Taiwán. Pharm Care Esp, 19(2), 58-68. Recuperado de https://www.researchgate.net/profile/Aldo-Alvarez-Risco2

Amibor, K C., \& Okonta, C. (2019). Attitude and Practice of Community Pharmacists towards Pharmaceutical Care in Nigeria. J. Drug Delive Ther, 9(6-s), 164-171. https://dx.doi:10.22270/jddt.v9i6s.3786

Choque, E., Peñaranda, R., Sivila, Y., \& Vargas, J. (2019). Satisfacción percibida del servicio de farmacias comunitarias independientes de la Ciudad de Sucre. Rev. Bio Scientia, 2(4), 19-29. Recuperado de https://revistas.usfx.bo/index.php/bs/article/view/327

Choque, R. (2005). Comunicación y educación para la promoción de la salud. Recuperado de http://www.razonypalabra.org.mx/libros/libros/comyedusalud.pdf

Coca, J.J. (2018). La comunicación en el uso responsable de los medicamentos y la venta cruzada. (Tesis Doctoral). Universidad Complutense de Madrid. España.

Elías, I., García, A., Besga, R R., \& Rouco, M. (2019). Condiciones básicas estructurales para la implementación del seguimiento farmacoterapéutico en farmacias comunitarias cubanas. Rev Cub Farm, 52(4), 1-18. Recuperado de https://www.medigraphic.com/pdfs/revcubfar/rcf-2019/rcf194c.pdf

García, A., García, E., Díaz, M., \& González, A. (2016) Propuesta de indicadores de resultados y de recursos para farmacias comunitarias cubanas. Rev Cub Farm, 50(1). Recuperado de https://scielo.sld.cu/scielo.php?script=sci_arttext\&pid=S003475152016000100008

González, G. (2019). Comunicación en y para la promoción de salud. En González G. Comunicación en salud: Conceptos y herramientas. 1era Edición. Argentina. Editorial Universidad General Sarmientos, p12-25. Recuperado de https://www.researchgate.net/publication/336275667_Comunicacion_en_salud_ conceptos_y_herramientas/citation/download

Lemay, J., Waheedi, M., Al-Taweel, D., Bayoud, T., \& Moreau, P. (2018). Clinical pharmacy in Kuwait: Services, provided perceptions and barriers. Saudi Pharm. J, 26(4), 481-486. https://doi.org/10.1016/j.jps.2018.02.011

Lugo, G.B., Vera, Z.C., Aguilar, A., Samaniego, L.R., \& Maidana, G.M. (2019). Barreras que impiden la implementación efectiva de la Atención Farmacéutica. Ars Pharm, 60(4), 199-204. https://doi.org/10.30827/ars.v60i4.9403

Martín, A., \& Valles, E. (2014). Habilidades para la Atención Farmacéutica I. En: Taller sobre Comunicación al paciente. Educafarma, 59-61. Recuperado de https://gredos.usal.es/bitstream/handle/10366/124347/EducaFarma_2014_9_

Martínez, D.M. (2015). Impacto de la intervención farmacéutica de educación sanitaria en pacientes polimedicados mayores de 65 años en farmacias comunitarias. (Tesis 
Doctoral). Facultad de Ciencias de la Salud. Universidad Católica San Antonio. Murcia, España.

Mateu, L., Sedeño, C., Estrada, V., \& García, I. (2017). La gestión del conocimiento en los servicios farmacéuticos de La Habana. Rev Cub Farm, 51(4) Recuperado de http://www.revfarmacia.sld.cu/index.php/far/article/view/238/169

Ministerio de Educación Superior. (2017). Plan de estudio E: Carrera Ciencias Farmacéuticas. La Habana Cuba.

Ministerio de Salud Pública (2005) Manual de Normas y Procedimientos de la farmacia comunitaria.

Recuperado

de www.sld.cu/galerías/pdf/sitios/revfarmacia7manual_normas_y_procedimientos. _farmacia_comunitaria._2005.pdf

Pérez, B., García, V., Gastelurrutia, M., Varas, R., Sáez, L., Martínez, F., \& Benrimoj, S. (2014). Percepción de los formadores Colegiales sobre el futuro profesional de esta nueva figura laboral en la farmacia comunitaria. Pharm Care Esp, 16(2), 81-8. Recuperado www.pharmcareesp.com/index.php/PharmaCare/article/view/181

Pérez, C., \& Valdearcos, S. (2018). Percepción de los profesionales sanitarios del valor del Farmacéutico de Atención Primaria y de la necesidad de implantar un Servicio de Atención Farmacéutica. Pharm Care Esp, 20(2), 132-148. Recuperado de www.pharmcareesp.com/index.php/PharmaCare/article/view/430

Pérez, F.J., Mohedano, R.B., \& Hernando, MA. (2019). Estudio de la percepción de los profesionales implicados en el proceso de suministro de medicación a ancianos que viven en residencias desde una farmacia comunitaria. Farmacéuticos Comunitarios, 11(4), 49-55. https://doi:10.5672/FC.21739218.(2019/Vol11).004.05

Prasojo, SA., \& Suci, P.S.S. (2019). The empirical test of pharmacist-patient relationship model in hospital pharmacy practice: Indonesia context. Research J. Pharm. and Tec, 12(10), 4623-4627. Recuperado de https://rjptonline.org/AbstractView.aspx?PID=2019-12-10-5

Reglamento General de Farmacias Comunitarias. (2016). Ministerio de Salud Pública de Cuba. Resolución 88/2016. Capítulo 13: Docencia, Capacitación e Investigación, artículos: 90-92,18-19.

Roig, J.C., Linares, M., Mejías, M., Pascual, M., Villar, I., \& Vitoria, G. (2012). Recursos necesarios para la dispensación de medicamentos y productos sanitarios complejos en la farmacia comunitaria. Farmacéuticos Comunitarios,4(Suplemento1). Recuperado de http://www.pdcorynthia.sld.cu/Documentos/biblioteca/dispensarizacion.pdf

Sabarte, M. (2016). Interacción farmacéutico-paciente en la farmacia comunitaria, en particular, con el proceso y aplicación de SFT (Tesis Doctoral) Facultad de Farmacia. Universidad de Granada. España

Sabater, M., Fernández, F., Sabater, D. Martínez, F., \& Isacc, S. (2016). Heathcare professional-patient relationships: Sistematic review of theoretical from a community pharmacy perspective. Patient Education and Counseling, 99, 339-347. Recuperado de https://pubmed.ncbi.nlm.nih.gov/26475728/

Saffouh, M., Sohil, H., \& Khaja, M. (2016). Qatar. Pharmacists' understanding, attitudes, practice and perceived barriers related to providing pharmaceutical care. Int J Clin Pharm, 38(2), 330-43. https://doi:10.1007/s11096-016-0246-0 
Sedeño, C., \& Sánchez, C. (2020). Sobre la historia de la farmacia comunitaria. Rev Cub Farm, $52(4)$

Recuperado

de http://www.revfarmacia.sld.cu/index.php/far/article/view/4

Suárez, Y. (2017). Nuevo plan de estudio para la Licenciatura en Ciencias Farmacéuticas. Rev Cub Farm, 51(2). Recuperado de http://www.revfarmacia.sld.cu/index.php/far/article/view/223/115

Valles, R., Aranzana, F., Puig, R., Ponsa, M., \& Ballart, F. (2018). Programa de colaboración entre la Farmacia de Atención Primaria y la Farmacia Comunitaria del Colegio de Farmacéuticos de Barcelona: resumen del documento de consenso. Pharm Care Esp, 20(2), 96-114. Recuperado de www.pharmcareesp.com/index.php/PharmaCare/article/view/413

Ventocilla, F.A. (2015). Percepción del paciente sobre el cuidado humanizado que brinda el profesional de enfermería en el Servicio de Observación del Hospital de Emergencia Casimiro Ulloa 2015. (Tesis Especialización). Universidad Privada Arzobispo Loay. Recuperado de https://core.ac.uk/download/pdf/323348929.pdf

Wells, K.M, Thornley, T, Boyd, M.J, \& Boardman, H.F. (2014). Views and experiences of community pharmacists and superintendent pharmacists regarding the New Medicine Service in England prior to implementation. Res Social Adm Pharm, 10(1), 58-71. https://doi:10.1016/j.sapharm.2013.03.003 